\title{
REPRODUÇÃO, DESENVOLVIMENTO EMBRIO-LARVAL E TESTES PRELIMINARES DE TOXICIDADE DE Parablennius pilicornis (CUVIER, 1829)(PISCES: BLENNIIDAE).*
}

\author{
RESGALLA Jr., C. $;$ MORELLI, F. ${ }^{1}$, RODRIGUES-RIBEIRO, M. ${ }^{1}$ \& A. BRANDELLI ${ }^{2}$ \\ ${ }^{1}$ CTTMar/UNIVALI, Cx.P. 360, Itajaí, SC - 88302-202 \\ ${ }^{2}$ BIOENSAIOS Consultoria Ambiental - Zamenhoff, 184, Porto Alegre, RS - 90550-090
}

\begin{abstract}
RESUMO
Parablennius pilicornis é uma espécie de peixe marinho comum no litoral brasileiro, apresentando ampla distribuição e caracterizada por ser o primeiro, entre os vertebrados, a colonizar substratos artificiais. Devido ao fato dos adultos apresentarem adaptação em laboratório, tamanho adequado, alta atividade reprodutiva, postura demersal além de sua disponibilidade no ambiente ao longo de todo o ano, fazem desta uma espécie com potenciais para a sua aplicação em testes toxicológicos. Este manuscrito apresenta os resultados preliminares sobre alguns aspectos reprodutivos, desenvolvimento embrio-larval, e testes toxicológicos da fase embrio-larval com um químico de referência a partir do cultivo destes peixes em laboratório. Por um período de 6 meses foram mantidos 8 casais de P. pilicornis, alimentados diariamente com adultos de Artemia sp congelados, renovação parcial de água e observação de posturas. Das 38 posturas obtidas, o número médio de ovos foi de 137,2, onde o tempo de desenvolvimento variou entre 8 e 20, dias dependendo da temperatura média de incubação $\left(25\right.$ a $\left.18^{\circ} \mathrm{C}\right)$. Os estágios de desenvolvimento do embrião e a larva recém-eclodida foram descritos mediante o acompanhamento de uma desova determinada. Testes de toxicidade embrio-larval, semi-estáticos com uma duração máxima de 15 dias, utilizando-se o químico de referência cloroacetamida, permitiram determinar a concentração incipiente de 25 e $20 \mathrm{mg} / \mathrm{l}$ ( $\mathrm{Cl}$ 25, concentração de inibição de $25 \%$ ) para as temperaturas de 22 e $25^{\circ} \mathrm{C}$ respectivamente.
\end{abstract}

Palavras chave: Blenniidae, Desenvolvimento Embrio-larval, Testes Toxicológicos.

\section{REPRODUCTION, EMBRYO-LARVAL DEVELOPMENT AND PRELIMINARY TOXICOLOGICAL TEST WITH Parablennius pilicornis (CUVIER, 1829)(PISCES: BLENNIIDAE).}

\begin{abstract}
Parablennius pilicornis is a common marine fish widely distributed in the Brazilian coast, and it is characteristic to be the first invertebrate to colonize the artificial substratum. This specie shows many aspects suitable for its use in toxicological tests, such as: resistance to laboratory conditions, adequate size, high reproductive activity, demersal eggs, and occurence along the year in the environment. This paper presents the first results about the aspects of reproduction, embryolarval development and toxicological tests with a reference toxicant. During 6 mouths, 8 couples of P. pilicornis were maintained in laboratory conditions, feeded with frozen Artemia sp adults, partial water renovation and monitored for the occurence bearing on empty oysters shell. A total of the 38 bearing were observed with an average eclosion succes of $72 \%$. The average number of eggs per
\end{abstract}

* Trabalho apresentado no III Simpósio sobre Oceanografia - IOUSP, 02 a 06 de Dezembro de 1996. 
RESGALLA Jr. et al.: Reprodução e testes de toxicidade de Parablennius pilicornis.

bearing were 137.2, where eggs development time ranged between 8 and 20 days and was function of the incubation temperature $\left(25\right.$ to $\left.18^{\circ} \mathrm{C}\right)$. The development stages of the embryos and hatchlings were described by following a bearing. Semi-static toxicological tests for embryo-larval stage, with the reference toxicant chloroacetamide, showed an IC25 threshold of 25 and $20 \mathrm{ml} / \mathrm{l}$ at 22 and $25^{\circ}$ $\mathrm{C}$ respectively, for 15 days of the time exposure.

Key-words: Blennidae, Embryo-larval Development, Toxicological Test.

\section{INTRODUÇÃO}

As espécies de peixes marinhos da família Blenniidae são caracterizadas por uma alta abundância em ambientes costeiros, incluindo recifes rochosos, poças de maré e estruturas artificiais, estando sua densidade dependente da disponibilidade de macroporos que oferecem refúgio, condições de postura e alimento (Teixeira, 1992). Parablenius pilicornis é uma espécie comum a esta família (Fig. 1) e que apresenta uma ampla distribuição na costa leste americana, ocorrendo desde o Estado da Flórida nos Estados Unidos da América, até Santa Catarina no Brasil (Menezes \& Figueiredo, 1995).

Este pequeno peixe apresenta várias características que fazem dele uma espécie com grande potencial para aplicação em testes toxicológicos. P. pilicornis é importante ecologicamente nos ambientes costeiros, resistente a sua manutenção em laboratório, apresenta tamanho adequado (média de 53,2 mm para os machos e $42,5 \mathrm{~mm}$ para as fêmeas), possui alta atividade reprodutiva, postura demersal, e uma disponibilidade no ambiente ao longo de todo o ano. Somado a estes fatos, existe hoje uma grande necessidade em

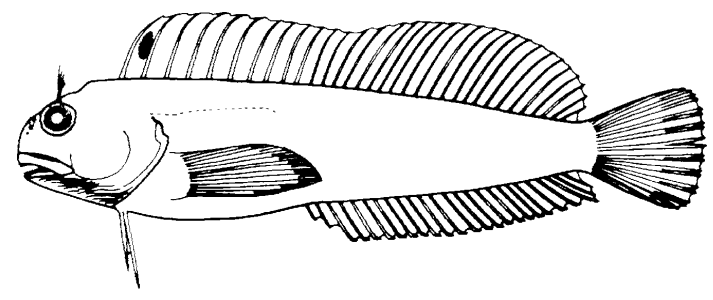

Figura 1 - Parablennius pilicornis (retirado de Menezes \& Figueiredo, 1995). buscar espécies de vertebrados nativos, para a utilização como organismos teste na avaliação dos impactos antropogênicos na costa brasileira.

No Brasil, estudos voltados para a investigação de vertebrados nativos aplicados como indicadores de poluição são limitados. Destacam-se, os estudos da CETESB com o Lambari (Hyphesobrium sp) e do Barrigudinho (Synolebias sp) desenvolvido pela UFRGS, sendo em ambos os casos, espécies de água doce. Para espécies marinhas, existem experimentos em andamento com juvenis de Tainha (Mugil sp) realizado pela UFPe, o Barrigudinho (Poecilla sp) estudado pela UFRJ e o Peixe Rei (Odonthestes sp) pela FURG. De acordo com os últimos encontros e congressos científicos nacionais sobre o tema, observa-se, para os ambientes marinhos, que as investigações quase sempre restringem-se a equinodermas, anfípodas, misidáceos e decápodas. Os laboratórios nacionais que realizam prestações de serviços especializaramse em testes de toxicidade restritos a peixes de água doce, que podem ser nativos para testes de toxicidade aguda (e.g. Brachidarieo rerio ou Paulistinha), ou exóticos para testes crônicos (e.g. Pimephales promelas, ou "Fathead Minnow").

Para a utilização de qualquer espécie como indicadora de poluição é necessário uma investigação de sua biologia. Estudos sobre $P$. pilicornis praticamente inexistem, ocorrendo entretanto trabalhos para outras espécies da mesma família, voltados principalmente aos aspectos etológicos, histológicos e reprodutivos (Almada \& Santos, 1995; Qasim, 1955; Santos, 1995; Santos et al., 1995 e Teixeira, 1992). 
Neste contexto, este manuscrito apresenta os resultados preliminares sobre alguns aspectos reprodutivos da espécie em laboratório, assim como alguns testes toxicológicos com a fase inicial de desenvolvimento frente a um químico de referência.

\section{MATERIAL E MÉTODOS}

Exemplares de $P$. pilicornis foram obtidos junto aos cultivos de mexilhões (Perna perna) e ostras (Crassostrea gigas) da Estação Experimental de Maricultura (Campus V CTTMar/UNIVALI), localizado no município de Penha, litoral norte de Santa Catarina. Estes peixes são encontrados em abundância nos espaços entre os moluscos, onde alimentamse da fauna acompanhante aos cultivos suspensos e utilizam conchas vazias como substratos para a realização de posturas, dos quais, os machos realizam o cuidado parental.

Por um período de seis meses foram mantidos oito casais de $P$. pilicornis em quatro aquários no laboratório de cultivo de organismos marinhos da CTTMar/UNIVALI. A rotina diária constou de sifonamento de $20 \%$ da água dos aquários, alimentação com adultos de Artemia sp congelados (produzidas nas salinas de Macau, Rio Grande do Norte) e observação da presença de postura no interior de conchas de ostras (Crassostrea gigas) vazias, oferecidas como substrato.

Quando observado a presença de desova, as conchas com os ovos foram transferidas e mantidas em frascos de 3 litros com água do mar filtrada (filtros Whatman GF/ F) a $33 \%$ (em média), com fotoperíodo de 10 horas de luz (d) e 14 horas de escuro (n) e forte aeração.

Foram estimados o tempo de desenvolvimento do ovo até sua eclosão e intervalo de eclosão (tempo em dias de eclosão entre o primeiro e o último ovo) em uma mesma postura, em função com a temperatura de incubação. Os estágios de desenvolvimento do ovo até sua eclosão foram descritos pelo acom- panhamento e registro fotográfico de ovos de uma mesma postura durante sete dias, os quais foram incubados a temperatura de laboratório $\left(22,3 \pm 3,8^{\circ} \mathrm{C}\right)$, salinidade de $35 \%$ e fotoperíodo de 10d:14n.

Testes estáticos para determinar o tempo de sobrevivência de larvas recém-eclodidas em condições de inanição (starvation) e utilizando-se como alimento a espécie de fitoplâncton Isochrysis galbana e o rotífero Brachionus plicatilis, ambos em abundância, foram conduzidos em 46 câmaras de $300 \mathrm{ml}$, com água do mar natural filtrada, a $34 \%$ de salinidade, fotoperíodo de $10 \mathrm{~d}: 14 \mathrm{n}$, e à $20^{\circ} \mathrm{C}$ $\left( \pm 2,2^{\circ} \mathrm{C}\right)$ de temperatura.

Testes semi-estáticos do tipo embriolarval com o químico de referência cloroacetamida, foram executados em temperaturas de 22 e $25^{\circ} \mathrm{C}\left( \pm 2^{\circ} \mathrm{C}\right.$ ) e fotoperíodo de $12 d: 12 n$ em incubadora. Foram utilizadas concentrações de 0,$0 ; 1,25 ; 2,5 ; 5,0 ; 10,0 ; 20,0$ e 40,0 mg/l de cloroacetamida, cada qual com três réplicas com 15 ovos em frascos de vidro com $100 \mathrm{ml}$ de solução teste. Os experimentos foram conduzidos em água do mar reconstituída a $33 \%$ de salinidade. Com os percentuais de sobrevivência foram estimados o Cl 25 (Concentração de Inibição a 25 \%), corrigidas pelo controle segundo a rotina do programa ICp, versão 2 (EPA, 1991), e construídas curvas de toxicidade-tempo.

\section{RESULTADOS E DISCUSSÃO}

\section{Características das posturas}

Das 38 posturas registradas, o número médio de ovos por postura foi de 137,2 com mínimo de 5 e máximo de 735. O tempo de desenvolvimento do ovo variou entre 8 a 20 dias e o intervalo da primeira a última eclosão, em uma mesma postura, variou de 1 a 7 dias, dependendo da temperatura de incubação (18 a $25^{\circ} \mathrm{C}$ ). $\mathrm{O}$ intervalo de tempo entre posturas, em um mesmo aquári,o variou de 5 a 10 dias, com uma taxa média de eclosão de $72 \%$. 
A figura 2 mostra os resultados de incubações em diferentes temperaturas, indicando uma relação inversa com o tempo de desenvolvimento e direta com o intervalo de eclosão. Estes resultados estão de acordo com estudos energéticos do metabolismo para os estágios iniciais de desenvolvimento de peixes segundo Kamler (1992).

\section{Desenvolvimento embrio-larval}

O comportamento reprodutivo dessa família, bem como o cuidado parental, os caracteres sexuais secundários e o desenvolvimento do embrião, já foram descritos por Qasim (1955) para a espécie Blennius pholis, apresentando considerável similaridade de hábitos observados para $P$. pilicornis, porém com grande diferença morfométrica. Tal como para B. pholis (Qasim, op. cit.), também foi observado, no ambiente e em laboratório, que o mesmo macho de $P$. pilicornis fecunda ovos de diferentes fêmeas. As desovas das distintas fêmeas fecundadas pelo mesmo macho são realizadas na mesma concha, levando a ocorrência de diferentes estágios de desenvolvimento dos ovos.

A partir de uma desova determinada, foram retirados da concha e fotografados dois ovos a cada dia, para documentar o desenvolvimento do embrião até sua eclosão. Tratando-se de uma espécie caracterizada por de- sovas demersais, associadas a fundos duros e tocas, o ovo recém depositado apesar de esférico, apresenta um disco basal de adesão com aspecto muco-filamentoso. Na mesma massa desovada, foram medidos 14 ovos, obtendo-se o diâmetro médio de $0,855 \mathrm{~mm}$ ( \pm $0,022 \mathrm{~mm}$ ), com um espaço perivitelínico de $0,125 \mathrm{~mm}( \pm 0,008 \mathrm{~mm})$.

O ovo fotografado no dia da desova já apresentava o desenvolvimento do pólo vegetativo, circundado por inúmeros glóbulos lipídicos e lipoproteicos, de tamanhos reduzidos, cuja coloração variava em tons de amarelo/dourado ao rosa/violeta (Fig. 3A). No segundo dia os glóbulos lipídicos mostravam uma clara migração ao pólo vegetativo, já bem visível, notando-se o processo de blastulação (Fig. 3B). Ao terceiro dia após a desova (Fig. $3 C)$, o embrião já mostrava-se bem diferenciado, em franco desenvolvimento, com os glóbulos de óleo todos bem aglomerados num extremo da massa de vitelo amarelada, agora circundada por duas faixas negras de melanóforos. Os ovos no quarto (Fig. 3D) e quinto dias (Fig. 3E) após a postura, não apresentaram diferenças aparentes entre si, excetuando-se a pigmentação dos olhos e a porcentagem de cauda livre do vitelo. Nestes estágios os embriões já encontravam-se bem definidos, com batimentos cardíacos e circulação sanguínea claramente evidenciados sob lupa. No quarto dia, encontrava-se preso ao

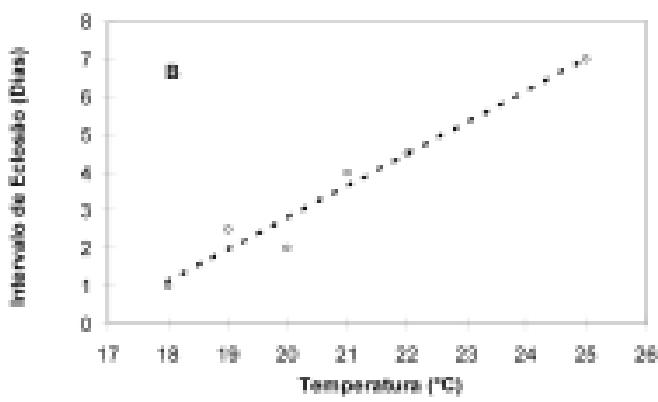

Figura 2 - A; Tempo médio de desenvolvimento dos ovos e B; Intervalo médio de eclosão em diferentes temperaturas de incubação. Os pontos representam as médias dos resultados obtidos e as linhas as suas tendências. 

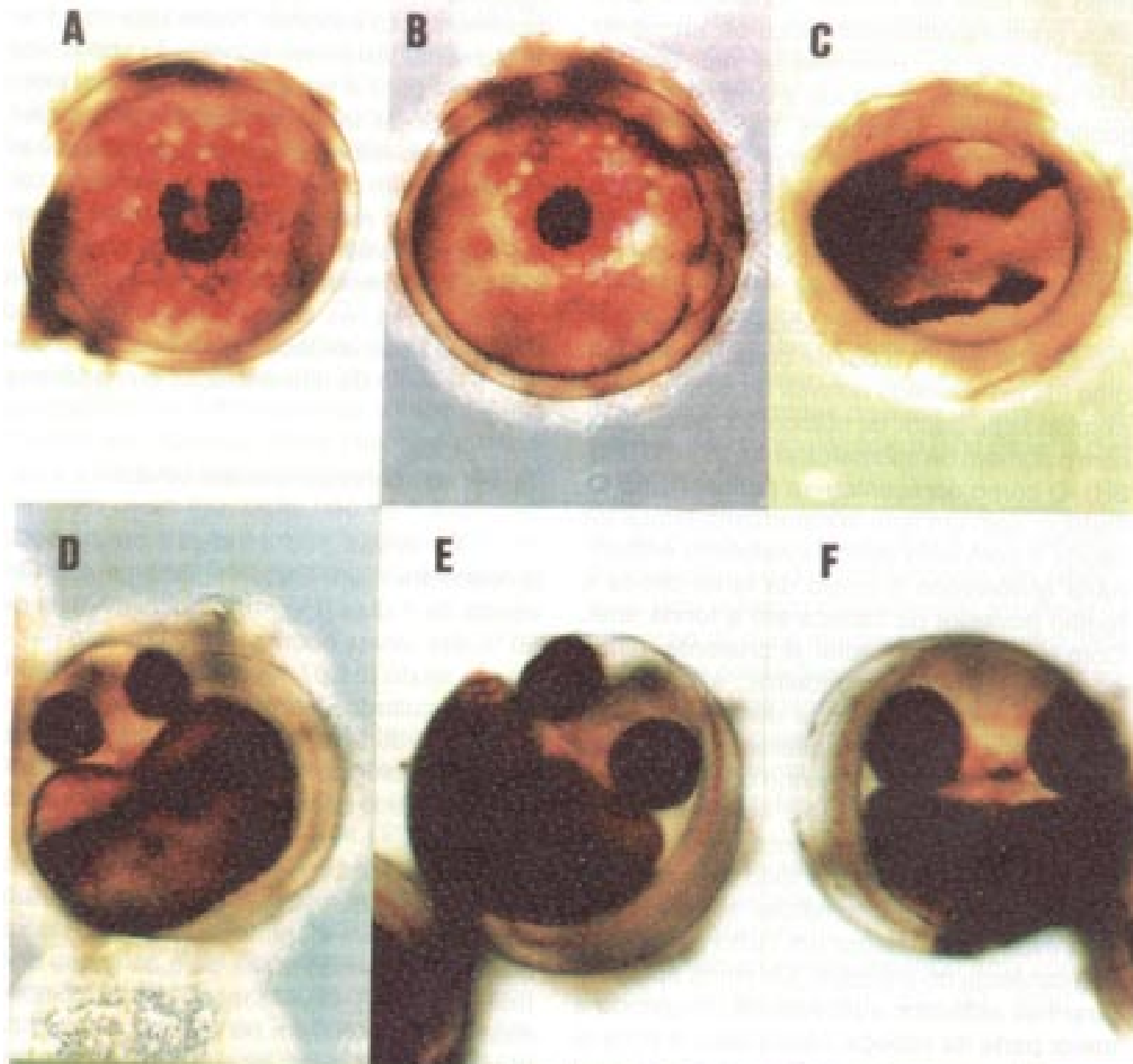

$\mathbf{E}$
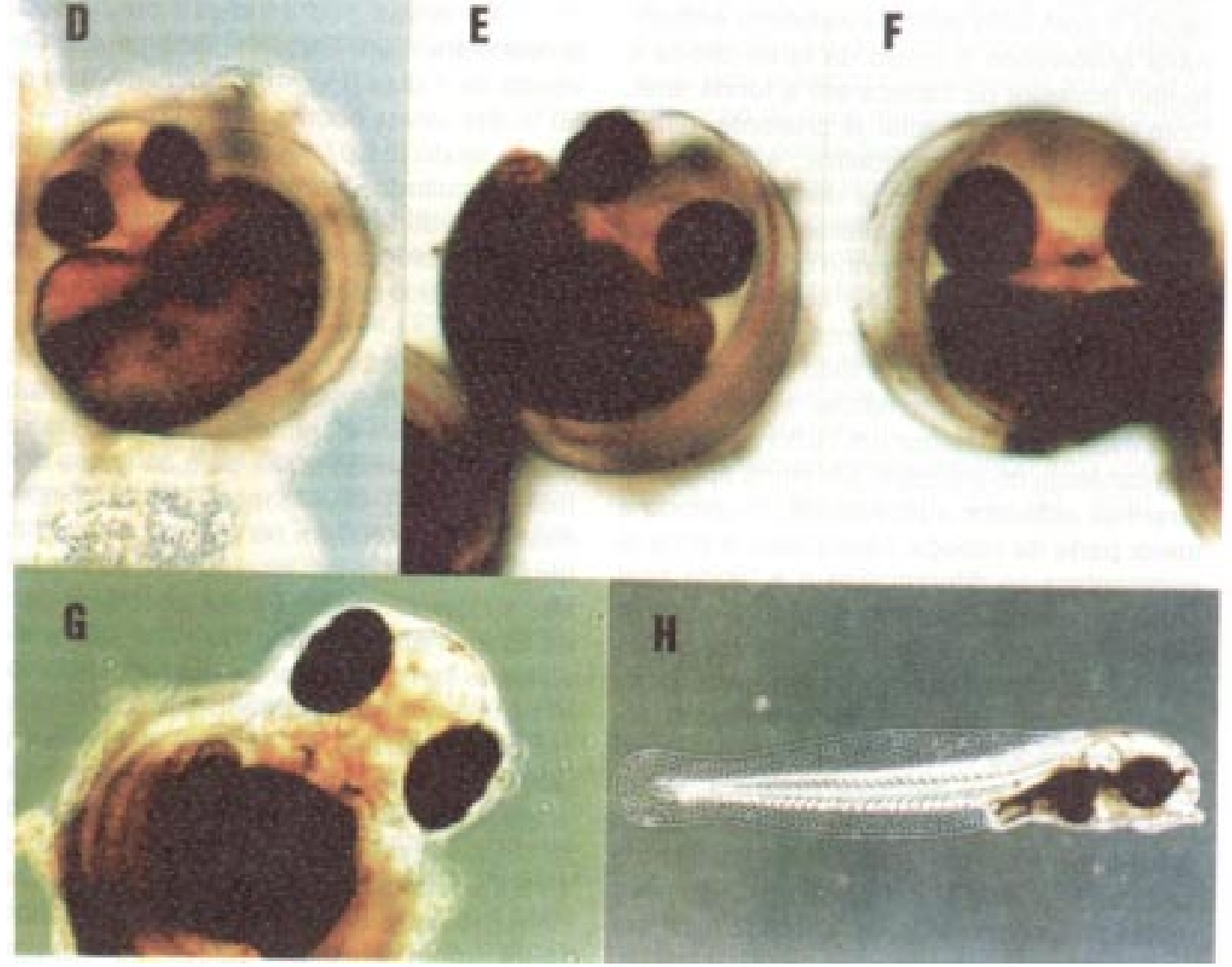

Figura 3 - Estágios de desenvolvimento do embrião com 24 horas de intervalo. Para detalhes vide texto. As fotos representam aumentos de 100X, exceto H que representa um aumento de 40X. 
vitelo em mais da metade do corpo e com olhos predominantemente escuros; enquanto que no quinto dia, mostrava-se mais livre, já com movimentos da cauda e com olhos predominantemente prateados. Da mesma forma, no sexto dia (Fig. 3F) o embrião encontrava-se com a cauda quase totalmente livre da massa de vitelo, com grande movimentação do corpo, eclodindo entre este e o sétimo dia (Fig. 3G) após a desova.

As larvas recém eclodidas mediram em média 3,09 mm $( \pm 0,06)$ de comprimento padrão (da extremidade anterior do focinho à extremidade posterior da notocorda, na cauda), com o número de miômeros entre 33 e 35 (Fig. $3 \mathrm{H})$. O corpo apresentou-se parcialmente cilíndrico, com um leve achatamento lateral da cauda e com uma película natatória embrionária envolvendo o corpo da larva desde a região posterior da cabeça até a fenda anal. Com exceção da peitoral já presente, ainda que sem os raios verdadeiros, a larva não apresentava formação das demais nadadeiras. A cabeça era bem diferenciada e volumosa em relação ao tamanho do corpo, ligeiramente inclinada para baixo, envolvendo uma massa vitelínica bem pigmentada e de pequeno tamanho, assim como todo o sistema digestivo. Estas características sugerem que esta espécie apresenta um curto período de permanência no plâncton. Os olhos apresentaram-se esféricos e projetados, ocupando a maior parte da cabeça. Nesta fase, a boca já apresentava-se diferenciada e a fenda anal pôde ser observada entre o sexto e o oitavo miômero.

Um aglomerado de melanóforos puntiformes a estrelados, ou ramificados, foram observados em ambos os flancos da cavidade abdominal da larva vitelínica. Outro padrão de pigmentação característico nesta fase esteve presente na região ventral do tronco e cauda da larva, com cerca de 25 grandes melanóforos estrelados, um em cada miômero a partir do oitavo ao décimo.

As larvas de comprimento padrão entre 3,50 a 4,00 mm já demonstram exauridas suas massas vitelínicas, iniciando o período de alimentação exógena. Nesta fase o padrão de pigmentação é mais acentuado, mantendo o descrito para a larva recém eclodida, além de apresentar um aglomerado de grandes melanóforos sobre as bases das nadadeiras peitorais, e um par na região posterior da cabeça, sobre o mesoencéfalo. Também a pigmentação nos flancos da cavidade abdominal parece incrementada. Com os comprimentos citados acima, as larvas já demonstram os primeiros raios verdadeiros da nadadeira peitoral e o início da diferenciação da nadadeira caudal.

\section{Testes de sobrevivência das larvas}

As larvas sob condição de inanição apresentaram um limite máximo de sobrevivência de 7 dias (Fig. 4). A sobrevivência de $50 \%$ das larvas ocorreu entre o quinto $(44,4$ $\%)$ e o sexto $(81,07 \%)$ dias após a eclosão. Estes resultados permitem avaliar o tempo máximo de duração de algum teste experimental (tanto fisiológico como de toxicidade) a partir da mortalidade observada nos exemplares do controle.

Nos frascos teste com alimento (Isochrysis galbana e Brachionus plicatilis), observou-se um incremento no tempo de sobrevivência, sendo maior para os testes com rotífero (Fig. 4), mas as mortalidades de $50 \%$ das larvas ocorreram no mesmo período de tempo dos testes em inanição. Estes resultados sugerem que uma dieta adequada para as larvas não foi alcançada, uma vez que o alimento sempre foi oferecido em excesso nos experimentos. Comparando-se as dietas oferecidas nos testes, acredita-se que as larvas pós-vitelínicas de $P$. pilicornis apresentam um limite crítico do tamanho do alimento, sendo nesta fase, predominantemente carnívoras. Estes resultados foram sugeridos pelos tamanhos médios das células de Isochrysis galbana (2 - $7 \mathrm{~mm})$, e do rotífero $(225 \mathrm{~mm})$ em comparação com a abertura máxima da boca das larvas $(215 \mathrm{~mm})$. Esta hipótese foi reforçada 
pela sobrevivência de 2 larvas por um período de 4 semanas em um frasco de incubação de ovos, onde ocorreu o desenvolvimento de uma população do copépodo bentônico do gênero Tisbia. Seguramente este pequeno sucesso foi conseguido pela disponibilidade de naupliu do copépoda $(<30 \mathrm{~mm})$, que serviram de alimento às larvas do Blenniidae.

Entretanto, a manutenção das larvas de Blenniidae com larvas trocófora do molusco bivalve Perna perna (61 - $72 \mathrm{~mm}$ ) e larvas pluteus do equinoderma Arbacia lixula $(75 \mathrm{~mm})$, que foram oferecidos em abundância, não apresentaram sobrevivência diferente aos frascos em inanição. Uma possível explicação a este fato estaria no valor energético do alimento oferecido, onde não só a abundância e o tamanho seriam os fatores principais as necessidades fisiológicas desta espécie de peixe (Kamler, 1992).

\section{Testes de toxicidade}

As curvas de toxicidade-tempo (Fig. 5) mostraram resultados muito próximos apesar da variação da temperatura. A curva para a temperatura de incubação de $22^{\circ} \mathrm{C}$ apresentou uma concentração incipiente de cloroacetamida de $25 \mathrm{mg} / \mathrm{l}$, enquanto que a $25^{\circ} \mathrm{C}$ a concentração foi de $20 \mathrm{mg} / \mathrm{l}$. Em ambos os casos, esta concentração foi atingida após o começo das eclosões nos frascos controle variando apenas 24 horas entre os testes realizados. Os pontos no final de cada curva teste refletem as mortalidades sofridas pelas larvas sob inanição, sendo mais prematuras no teste a $25^{\circ} \mathrm{C}$.

A análise diária de ovos e larvas nos frascos teste constou em avaliar o estado do organismo frente aos exemplares do controle. Ovos gorados (mudança de coloração do córiun ou ausência de pigmentação do embrião), eclosões prematuras (embrião vivo fora do ovo) e ainda larvas eclodidas com a cabeça presa no interior do ovo (uma eclosão de sucesso consta na saída inicial da cabeça da larva impulsionada pela cauda como mostra a figura $3 G$ ) foram consideradas efeitos adversos no teste. A grande maioria destes efeitos observados foi devido a influência do agente testado sobre o córiun do ovo, o que comprometeu sua resistência e sua função em proteger a viabilidade do embrião (Abel \& Axiak, 1991). Se isto for considerado, o tempo de duração do teste pode ser limitado até o início das eclosões obtidas nos frascos controle e que pode se estender por 3 dias. Este limite também foi confirmado pelas concentrações incipientes obtidas na figura 4. Assim, larvas livres devem ser registradas como sucesso e descartadas do teste, já que não sobrevivem à manipulação e à falta de uma dieta adequada.

Estes resultados refletem a necessidade de estudos para a melhoria dos métodos de manutenção dos progenitores em laboratório. Este esforço provavelmente resultaria na obtenção de ovos maiores, maior quantidade de vitelo e consequentemente uma maior sobrevivência das larvas (Kamler, 1992).

Como mencionado anteriormente, a espécie apresenta bom potencial de aproveitamento em testes de toxicidade, entretanto, os valores das concentrações incipientes obtidas foram elevadas, refletindo uma provável baixa sensibilidade desta espécie à substância de referência. Experimentos com Pimephales promelas, mostrou uma concentração letal (CL50) de $30 \mathrm{mg} / \mathrm{l}$ de

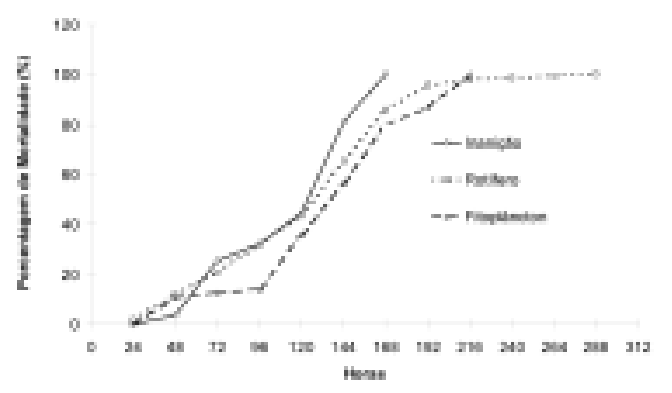

Figura 4 - Valores médios de mortalidade de larvas recém-eclodidas de $P$. pilicornis sob condição de inanição e em oferta de fitoplâncton e rotíferos como alimentos. 


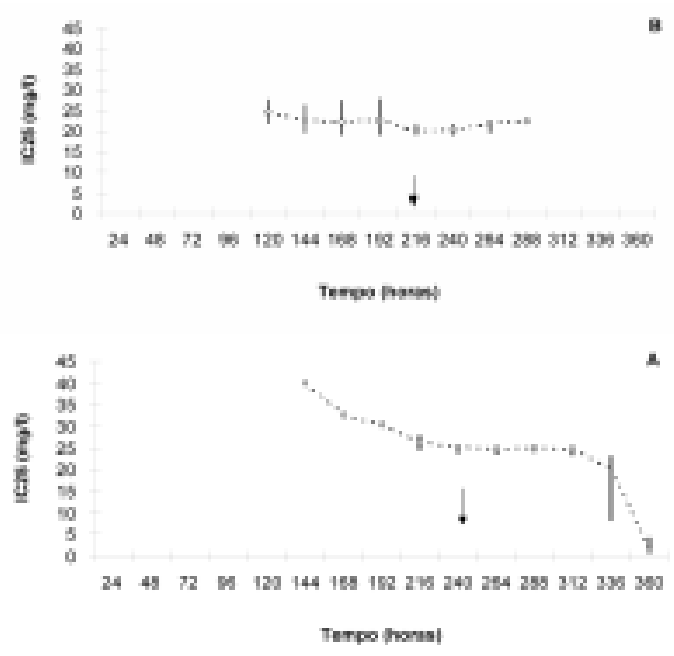

Figura 5 - Toxicidade-tempo para o químico de referência cloroacetamida em duas temperaturas de incubação. Cada ponto é representado pela média e intervalo de confiança de $95 \%$. As flechas indicam o primeiro dia de eclosão no controle. $\mathrm{A} ; 22^{\circ} \mathrm{C}$ e $\mathrm{B} ; 25^{\circ} \mathrm{C}$.

cloroacetamida em experimentos agudos (Brandelli, 1995). O desenvolvimento de testes em diferentes temperaturas e salinidades assim como o emprego de outros químicos de referência são passos necessários para uma possível padronização de um sistemateste baseado nesta espécie.

\section{RECOMENDAÇÕES}

Apesar das muitas vantagens que a espécie apresenta como organismo teste, existem ainda necessidades de investigações mais profundas sobre:

1. Condições de manutenção de adultos em laboratório (temperatura e alimento) buscando a produção de ovos maiores e com maior quantidade de vitelo, o que resultaria em larvas pós-vitelínicas mais resistentes.

2. A procura de alimento adequado para as larvas do Blennidae, aumentando suas taxas de sobrevivência para o período crítico no início da alimentação exógena.

3. Condições de manutenção de ovos e larvas nos frascos testes, envolvendo testes em fluxo contínuo ou em sistemas que permitam a manutenção das larvas em suspensão sem o seu gasto energético interno. Foi constatado que choques mecânicos proporcionados por aeração em ovos livres do substrato reduziram o intervalo de eclosão. Para as larvas, entretanto, estes choques aumentam sua mortalidade.

4. Testes de toxicidade com outras substâncias de referência e em diferentes condições laboratoriais (temperatura e salinidade principalmente).

5. E, finalmente, testes de sobrevivência das larvas e toxicológicos embrio-larval entre ovos obtidos em laboratório e no campo, buscando resultados que apontem para alguma variabilidade nos organismos teste.

\section{AGRADECIMENTOS}

Os autores agradecem ao apoio da equipe da Estação Experimental de Maricultura de Penha (Campus V - CTTMar/ UNIVALI) sem a qual não seria possível realizar todos os experimentos aqui apresentados.

\section{REFERÊNCIAS}

Abel, P.D. \& Axiak, V. 1991. Ecotoxicology and the Marine Environment. Ellis Horwood, New York, 265 pp.

Almada, V.C. \& R.S. Santos. 1995. Parental care in the rocky intertidal: a case study of adaptation and exaptation in Mediterranean and Atlantic Blennies. Rev. Fish Biol. Fish. 5:23-37.

Bradelli, A. 1995. Estudo da bioconcentração do fungicida Propiconazole em 
Pimephales promelas (Rafinesque, 1820). Tese de Mestrado, Univ. Fed. Rio Grande do Sul. 68pp.

EPA, 1991. Tecnical support document for water quality-based toxics control. US EPA Office Water. EPA-505-2-90-001.

Kamler, E. 1992. Early life history of fish. An energetics approach. Fish and Fisheries Series 4. Chapman \& Hall. London. 267pp. Menezes, N.A. \& J.L. Figueiredo. 1995. Manual de peixes marinhos do sudeste do Brasil. V Teleostei (4). Mus. Zoologia USP. 54-56.

Santos, R.S. 1995. Allopeternal care in the redlip blenny (Ophioblennius atlanticus
atlanticus)(Pisces: Blenniidae). J. Fish Biol. 47:76-88.

Santos, R.S.; Nash, R.D.M. \& S.J. Hawkins. 1995. Age, growth and sex ration of the Azorean rocky-pool Blenny, Parablennius sanguinolentus parvicornis. J. Mar. Biol. Ass. Uni. Kingdon. 75(3): 167-179.

Qasim, S.Z. 1955. The spawning and embryonic development of the Shanny (Blennius pholis L.). Proc. Zoo. London. 127:79-93.

Teixeira, R.L. 1992. Ocupação de um habitat artificial por Scartella cristata (Linnaeus, 1758)(Pisces: Blenniidae) em praia arenosa de Maceió (Alagoas-Brasil). Bol. Estud. Ciênc. Mar. 8:13-22. 